\title{
Application of a phase space beam position and size monitor for synchrotron radiation source characterization
}

\author{
N. Samadi $\odot,{ }^{1, *}$ X. Shi $\odot,{ }^{2}$ L. Dallin, ${ }^{3}$ and D. Chapman ${ }^{3,4}$ \\ ${ }^{1}$ Physics and Engineering Physics, University of Saskatchewan, \\ 116 Science Place, Saskatoon, Saskatchewan, S7N5E2, Canada \\ ${ }^{2}$ Advanced Photon Source, Argonne National Laboratory, \\ 9700 South Cass Avenue, Lemont, Illinois 60439, USA \\ ${ }^{3}$ Canadian Light Source, 44 Innovation Boulevard, Saskatoon, Saskatchewan, S7N2V3, Canada \\ ${ }^{4}$ Anatomy, Physiology and Pharmacology, University of Saskatchewan, \\ 107 Wiggins Road, Saskatoon, Saskatoon S7N 5E5, Canada
}

(Received 27 June 2019; published 12 December 2019)

\begin{abstract}
A phase space beam position and size monitor (ps-BPM) system was introduced previously to measure the electron source vertical position and angular motion along with the vertical source size and angular size at a single location in a synchrotron bend magnet beam line. The system was validated experimentally at the Canadian Light Source and demonstrated by a simulation that it is suitable for low-emittance light sources. In this work, the potential practicality of the ps-BPM system is explored with real-case studies. By combining the analysis in the time and frequency domain, information on the beam motion and size can be extracted and identified from both the source and the beam-line optics. Applications of the ps-BPM system at the Canadian Light Source are demonstrated by studying beam vibrations and machine insertion device field changes. Results of these studies provide guidelines for implementing a dedicated ps-BPM system at existing and planned synchrotron facilites.
\end{abstract}

DOI: $10.1103 /$ PhysRevAccelBeams.22.122802

\section{INTRODUCTION}

Accurate measurements of the electron source size and divergence are becoming increasingly important at synchrotron facilities as the next-generation light sources are being built with the goal of achieving the smallest possible emittance $[1,2]$.

Currently available ways of measuring the source size are relying on direct imaging or interference-based techniques. Direct imaging methods include pinhole imaging [3,4], imaging with Kirkpatrick-Baez mirrors [5], compound refractive lenses [6], and Fresnel zone plates [7,8]. The interferometry-based systems include double-slit diffraction [9-11], grating interferometry [12,13], and $\pi$ polarization [14].

In addition to the importance of measuring the source size at these next-generation light sources, beam stability is also of great concern. Real-time measurements of the position and angular position of the electron beam at a single location will be a powerful tool as a monitor and a diagnostic element and in a feedback system.

\footnotetext{
*Nazanin.samadi@usask.ca

Published by the American Physical Society under the terms of the Creative Commons Attribution 4.0 International license. Further distribution of this work must maintain attribution to the author(s) and the published article's title, journal citation, and DOI.
}

A phase space beam position and size monitor (ps-BPM) system was introduced previously $[15,16]$, that measures, in real time, all four quantities (size, divergence, position, and angular position) of the electron source in the vertical plane from a single measurement. The system was predicated to be able to measure source properties of fourthgeneration light sources with ultrasmall emittance [17].

As a newly developed system, the ps-BPM system differs from all other existing methods in terms of physical model and experimental details. Even though the demonstration and physical interpretation of the system have been reported, the study of its practical realization and real-case applications are still of great importance for considering its implementation at other facilities. In this work, applications of the ps-BPM system in studying the beam performance are demonstrated with experimental results at the Canadian Light Source (CLS).

\section{II. pS-BPM SYSTEM}

The ps-BPM system was described in detail in Refs. [15,18], and the physical model and key equations are summarized here.

The ps-BPM system uses the nearly monochromatic photon beam provided by a crystal monochromator around an absorption $K$ edge of a selected filter element (see Fig. 1). The monochromator can be of a reflection type 
(a)

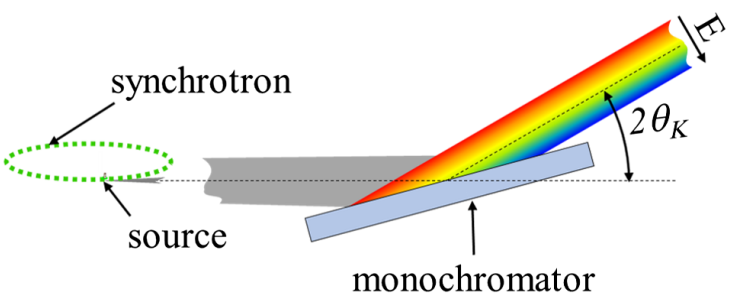

(b) Beam Side

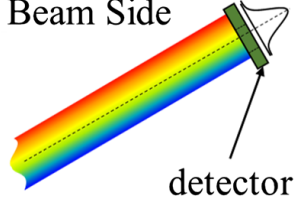

(c) Edge Side

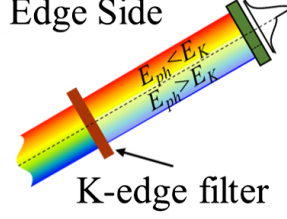

FIG. 1. Schematic of the ps-BPM system containing (a) the crystal monochromator, the $K$-edge filter, and the detector. A singlecrystal Bragg geometry is shown. (b) and (c) show the unfiltered beam size and the filtered edge side of the beam, respectively.

(Bragg geometry) or transmission type (Laue geometry). Half of the horizontal size of the photon beam is covered by the $K$-edge filter [Fig. 1(c), edge side], and the other half has no filter in the way [Fig. 1(b), beam side]. Both halves of the beam are then imaged with an area detector.

The unfiltered beam side of the image data is summed over a horizontal width, and the beam profile is shown just next to the detector symbol in Fig. 1(b) and in Fig. 2(a). This profile is fit with a Gaussian function from which the center location of the photon beam, $y_{\text {beam }}$, and the Gaussian distribution width $\sigma_{\text {beam }}$ is obtained.

For the filtered beam side, the image data are also summed over a horizontal width as shown in Figs. 1(c) and 2(b). This profile is then normalized by the unfiltered beam. The negative logarithm of this normalized filtered beam profile is taken to convert the filtered data to an absorption profile which then resembles the step function associated with the $K$ edge of the element as shown in Fig. 2(c). A spatial derivative is taken of this step function forming a peak that is then fit by a Gaussian function from

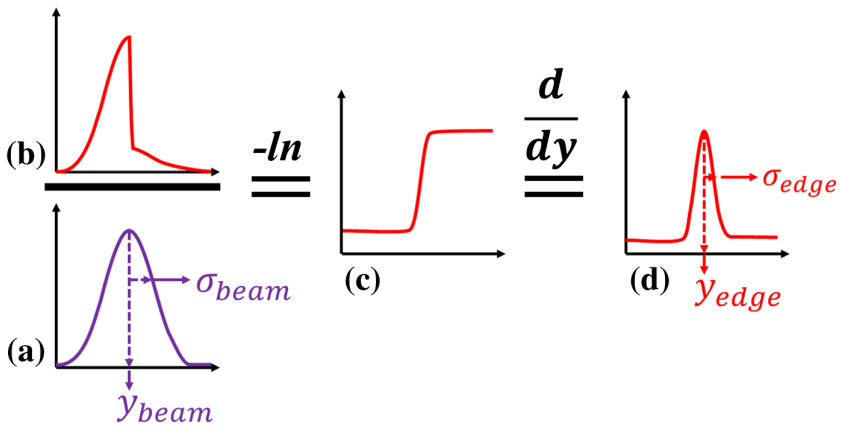

FIG. 2. Beam and edge analysis. (a) Beam profile along with Gaussian fit parameters. (b) Edge profile which is then normalized by (a). The negative logarithm of this normalized profile is shown in (c). The spatial derivative of the profile in (c) results in a peak shown in (d) along with its Gaussian fit parameters.

which gives an edge location $y_{\text {edge }}$ and width $\sigma_{\text {edge }}$, as shown in Fig. 2(d).

\section{A. Source position and angle measurements}

Referring to Fig. 1, when the electron source moves, the detected photon beam on the beam and edge sides may behave differently. For example, if the electron source has a fixed position but a changing angle, the detected photon beam on the beam side will move vertically according to the angle and the distance the detector is from the source point. However, on the edge side, the edge location will not move, as the monochromator will select out the same energy from the angular distribution of the source. This is because the angle of the photon beam to the monochromator crystal is directly related to the energies diffracted by the monochromator by Bragg's law.

Now if the electron beam is fixed in angle but moves vertically in position, the photon beam on the beam side will also move vertically, as will the edge location on the edge side. Thus, there is a simple relationship between the vertical angle and position of the electron beam to the measured photon beam and edge locations. This then can be used to determine, independently, the vertical position and angle of the electron source [15].

As shown graphically in Fig. 2(d), the center of the Gaussian fit to the filtered $K$-edge side $\left(y_{\text {edge }}\right)$ is a direct measure of the electron source position $y$ as

$$
y=y_{\text {edge }} .
$$

The unfiltered beam side of the data includes the position $y$ and angular position $y^{\prime}$ of the electron source according to

$$
y_{\text {beam }}=y+D y^{\prime},
$$

where $D$ is the source-to-detector distance.

By having the two measurements at the same time with the ps-BPM system, we can solve for the angular position of the electron source, $y^{\prime}$, based on Eqs. (1) and (2) as

$$
y^{\prime}=\left(y_{\text {beam }}-y_{\text {edge }}\right) / D .
$$

\section{B. Source size and divergence measurements}

We assume that the source size and divergence will be Gaussian distributions along with other factors that may contribute to the measured widths arising from the optics, the filter material, and the natural opening angle of the photon beam from the source. The spatial width $\sigma_{\text {edge }}$ of the Gaussian fit from the filtered $K$-edge side of the photon beam includes contributions from the natural width of the $K$-edge filter element $\sigma_{y_{K \text {-edge }}^{\prime}}$, the angular acceptance of the monochromator $\sigma_{y_{\text {mono }}^{\prime}}$, and the vertical size of the electron source $\sigma_{y}$, all added in quadrature as 


$$
\sigma_{\text {edge }}^{2}=\sigma_{y}^{2}+\left(D \sigma_{y_{K \text {-edge }}^{\prime}}\right)^{2}+\left(D \sigma_{y_{\text {mono }}^{\prime}}\right)^{2} .
$$

The electron source size $\sigma_{y}$ is then

$$
\sigma_{y}=\sqrt{\sigma_{\text {edge }}^{2}-\left(D \sigma_{y_{\text {Kedge }}^{\prime}}\right)^{2}-\left(D \sigma_{y_{\text {mono }}^{\prime}}\right)^{2}} .
$$

The measured spatial width $\sigma_{\text {beam }}$ of the unfiltered side is the combination of the electron source size $\sigma_{y}$, divergence $\sigma_{y^{\prime}}$, and the opening angle of the photon beam $\sigma_{y_{\mathrm{Ph}}^{\prime}}$, added in quadrature as

$$
\sigma_{\text {beam }}^{2}=\sigma_{y}^{2}+\left(D \sigma_{y^{\prime}}\right)^{2}+\left(D \sigma_{y_{\mathrm{Ph}}^{\prime}}\right)^{2} .
$$

By having the opening angle of the photon beam, modeled as a Gaussian, ${ }^{1}$ and the measured $\sigma_{y}$ from the edge side, we can solve for the divergence of the electron source as

$$
\sigma_{y^{\prime}}=\frac{1}{D} \sqrt{\sigma_{\text {beam }}^{2}-\sigma_{y}^{2}-\left(D \sigma_{y_{\mathrm{Ph}}^{\prime}}\right)^{2}} .
$$

This shows by having measurements of the unfiltered beam side and the filtered $K$-edge side we can measure the vertical position, angle, size, and divergence of the electron source at the same time. However, to arrive at these values, the contributions from $\sigma_{y_{K \text {-edge }}^{\prime}}, \sigma_{y_{\text {mono }}^{\prime}}$, and $\sigma_{y_{\mathrm{Ph}}^{\prime}}$ need to be determined [18]. The $\sigma_{y_{K \text {-edge }}^{\prime}}$ derives from the tabulated energy width of the $K$-edge filter element [19]. The $\sigma_{y_{\text {mono }}^{\prime}}$ can be calculated based on the crystal material, reflection, and photon energy [20]. The photon angular distribution can be calculated and then fit with a Gaussian to arrive at $\sigma_{y_{\mathrm{Ph}}^{\prime}}[21]$.

\section{APPLICATIONS OF THE ps-BPM SYSTEM}

The application of the ps-BPM system is demonstrated below to show the ability to provide useful information regarding the source and even beam-line properties. The merit of the system to distinguish and measure multiple electron source properties implies that special data treatment and analysis are necessary to take full advantage of the system. In Sec. III A, we first show typical outputs of the psBPM system and how to combine the time and frequency domain analysis to extract source and optics information. Section III B focuses on the beam vibration analysis. Finally, Sec. III C shows a real-case machine study.

\section{A. Typical measurements}

Measurements using the ps-BPM system are shown to demonstrate the full characterization of the electron beam in the vertical direction during normal operations of the

\footnotetext{
${ }^{1}$ The Gaussian distribution assumption applies when the photon energy of the selected beam is above the critical energy of the bend magnet.
}

CLS facility. The measurements were performed at the Biomedical Imaging and Therapy (BMIT) bend magnet (BM) beam line [22-24] at the CLS. The photon energy was tuned to the barium $K$ edge at $37.441 \mathrm{keV}$ by the Si (220) double crystal monochromator (DCM). The projected concentration of the barium filter is $35 \mathrm{mg} \mathrm{cm}^{-2}$. The images of the filtered and unfiltered photon beam were recorded by a flat panel Hamamatsu detector (Hamamatsu Photonics, Hamamatsu City, Shizuoka Prefecture, Japan) at $D=20 \mathrm{~m}$ from the BM source. The detector pixel size was square with a linear dimension of $100 \mu \mathrm{m}$. A total of 3000 images were taken with an acquisition time of $0.03 \mathrm{~s}$ for each image.

The measurement results are shown in Fig. 3. The beam positions, including the source position $y$, angular position projected at the detector location, $D y^{\prime}$, and their combined position $y_{\text {beam }}=y+D y^{\prime}$, are shown as a function of the time in Fig. 3(a). The numbers at the tops in Figs. 3(a) and 3(c) are the standard deviation of the three positions over the entire measurement period of $90 \mathrm{~s}$. The standard deviation values are the direct evaluation of the amplitude of beam motion. At the detector location, the angular motion of the source has a larger effect than the source position motion.

The power spectral distribution (PSD) function, obtained from the Fourier transform of the time-dependent signal, allows characterizing the measured beam position in the frequency domain. The PSD functions of the three curves in Fig. 3(a) are shown in Fig. 3(b). With the sampling rate of $33 \mathrm{~Hz}$, positional variations or vibrations up to $16.7 \mathrm{~Hz}$ can be detected.

The total beam motion $y+D y^{\prime}$ at the detector location in the right panel in Fig. 3(b) shows three characteristic peaks at $6.7,14.2$, and $14.9 \mathrm{~Hz}$, which are clearly from the angular motion as seen in the middle panel in Fig. 3(b). The small frequency band around the $12.2 \mathrm{~Hz}$ peak, however, shows up in both the source position and angular position but not in the combined beam position on the detector.

The cause of the $12.2 \mathrm{~Hz}$ peak is the vibration of the DCM in the overall pitch angle, where both crystals rotate together (see Sec. III B for details). Because of the fixed axis geometry of the DCM, the beam position at the detector will not change with the DCM vibration. However, the Bragg angle change alters the photon energy center, which is interpreted by the ps-BPM system as the source position move. On one hand, it means that the monochromator used in the ps-BPM system needs to be stable or calibrated with vibration analysis. On the other hand, it shows the possibility of using the system to identify the optics vibration along the beam line.

The beam sizes, including the source size $\sigma_{y}$, angular distribution projected on the detector, $D \sigma_{y^{\prime}}$, and the total beam size $\sigma_{\text {beam }}$, are shown as a function of the time in Fig. 3(c). All curves are offset to their own average values, which are indicated in the figure. The light colored curves 

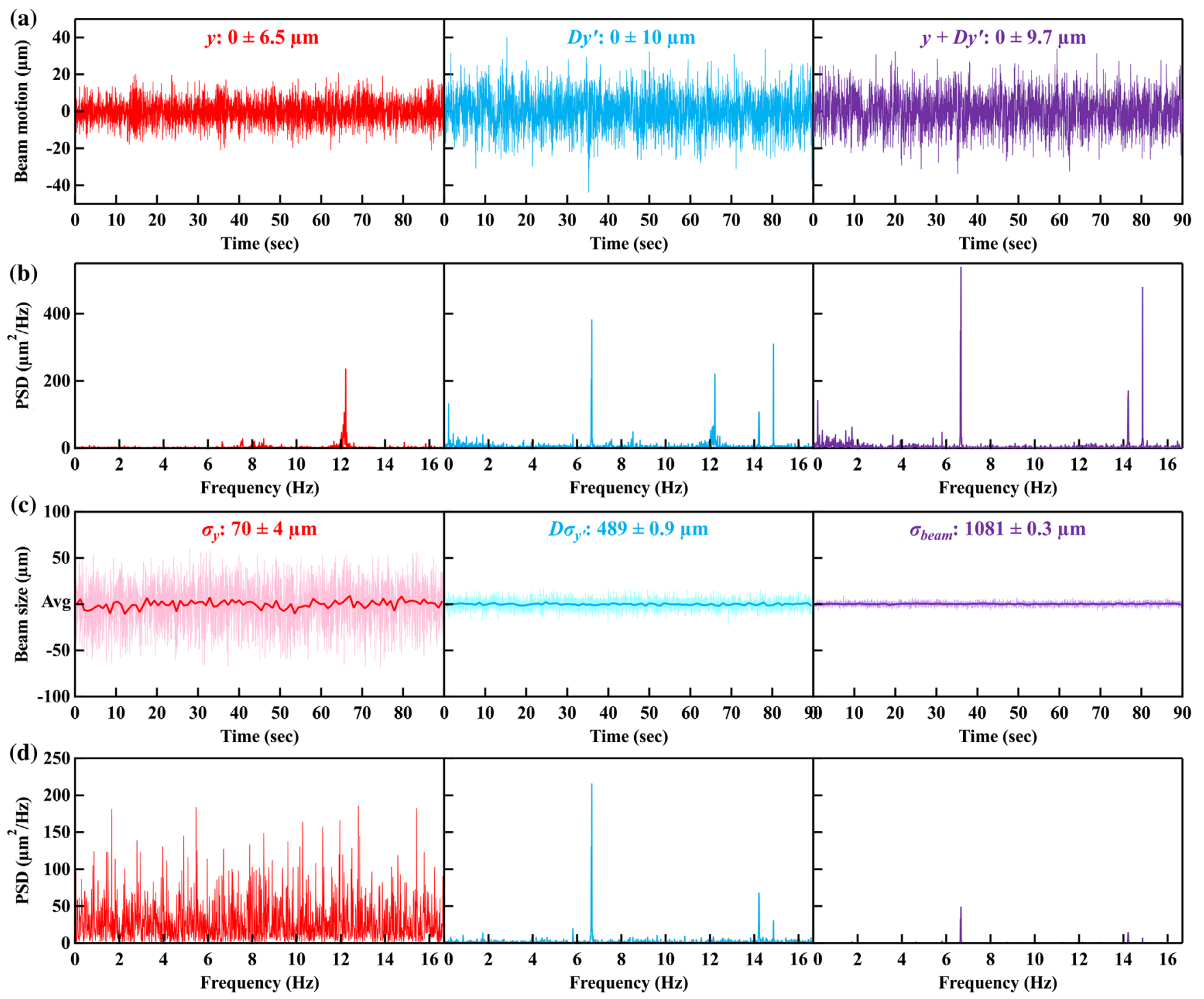

FIG. 3. (a) Beam motions including $y$ (left), $D y^{\prime}$ (middle), and $y+D y^{\prime}$ (right) as a function of the time. (b) PSD functions of the three curves in (a). (c) Time evolution of $\sigma_{y}$ (left), $D \sigma_{y^{\prime}}$ (middle), and $\sigma_{\text {beam }}$ (right). The light colored and dark colored curves are for a sampling time of 0.03 and $0.9 \mathrm{~s}$, respectively. (d) PSD functions of the light colored curves in (c).

are plotted in the original timescale ( $0.03 \mathrm{~s}$ steps). Since the beam size measurements are photon hungry, a longer acquisition time is needed to improve sensitivity [18]. In Fig. 3(c), the overplotted curves with dark colors were obtained by averaging every 30 points $(0.9 \mathrm{~s})$. The standard deviation values shown in the figure are also for the averaged curves, which show the sensitivity of the system for each of the three parameters. For the setup at the CLS BMIT beam line, an integration of $0.9 \mathrm{~s}$ is adequate to reach the sensitivity of $4 \mu \mathrm{m}$ for source size measurement.

The corresponding PSD functions of the fast measurement data [light colored curves in Fig. 3(c)] are shown in Fig. 3(d). The PSD function for $\sigma_{y}$ shows only random noise, indicating that the present system is not fast enough to monitor source size changes on the millisecond scale. But, the PSD functions for $D \sigma_{y^{\prime}}$ and $\sigma_{\text {beam }}$ show clear frequency peaks that are consistent with the ones observed in the position plots [see Fig. 3(b)], which is an indication of adequate sensitivity.

By combining real-time measurement and frequency analysis, the ps-BPM system provides useful beam information. All results shown in Fig. 3 can be real-time outputs for a dedicated system at a synchrotron BM beam line. Optimization has been carried out to improve the sensitivity of the ps-BPM system for measuring the ultrasmall source at planned low-emittance storage rings [17]. Results here provide guidelines for building the practical system and the associated control and data acquisition procedures.

\section{B. Beam vibration analysis}

For a radiation-based source monitor, the stability of optical components is extremely important, as the system 

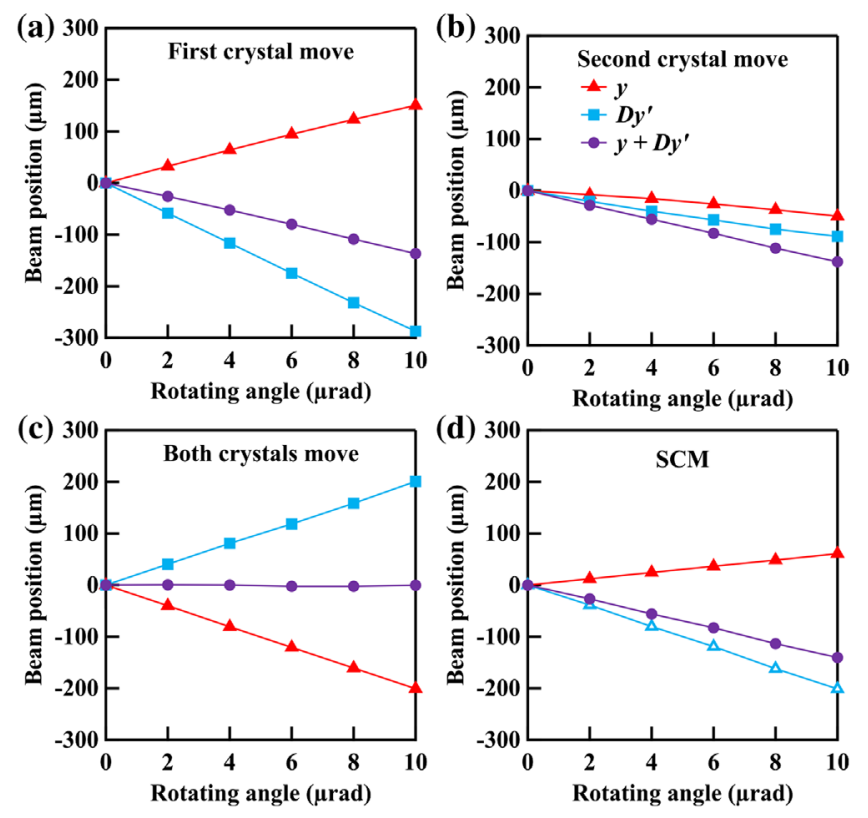

FIG. 4. Simulated beam position response as a function of the rotation angle of (a) the first crystal, (b) the second crystal, (c) both crystals of the DCM, and (d) the SCM crystal. The markers represent the extracted source position $y$ (triangles), projected source angle $D y^{\prime}$ (squares), and the total beam position $y+D y^{\prime}$. The curves provide a visual aid.

cannot distinguish contributions from the source and optics. It is thus crucial to either remove or identify system errors from the optical instability. Each optical component has characteristic eigenfrequencies which depend on its mechanical structure. There are also forced vibrations propagated from the floor and amplified through the support structure of the optics. These vibration sources could be identified and possibly mitigated with proper vibration testing using accelerometers and frequency analysis. If needed, the contribution of the system instability can then be removed from the data analysis using frequency filters.

To further understand the monochromator vibration, raytracing simulations were performed using ShadowOui [25] in the OASYS environment [26]. Simulation details can be found in Samadi et al. [18].

Figure 4 shows the beam position as a consequence of the input angular motion of the DCM crystals [Figs. 4(a)-4(c)] and single-crystal monochromator (SCM) [Fig. 4(d)]. Among all four cases, only Fig. 4(c) shows a zero total beam motion, $y+D y^{\prime}$ (solid circles). The source position $y$ (solid triangles) and angular position $D y^{\prime}$ (solid squares) have the same amplitude but opposite sign. This is consistent with the experimental observation shown in Fig. 3(a).

In all other three cases [Figs. 4(a), 4(b), and 4(d)], the total beam motion has the same amplitude, while $y$ and $D y^{\prime}$ vary in amplitude and sign. Thanks to the simultaneous measurement of $y$ and $D y^{\prime}$ by the ps-BPM system, the vibration source of the monochromator crystals can be
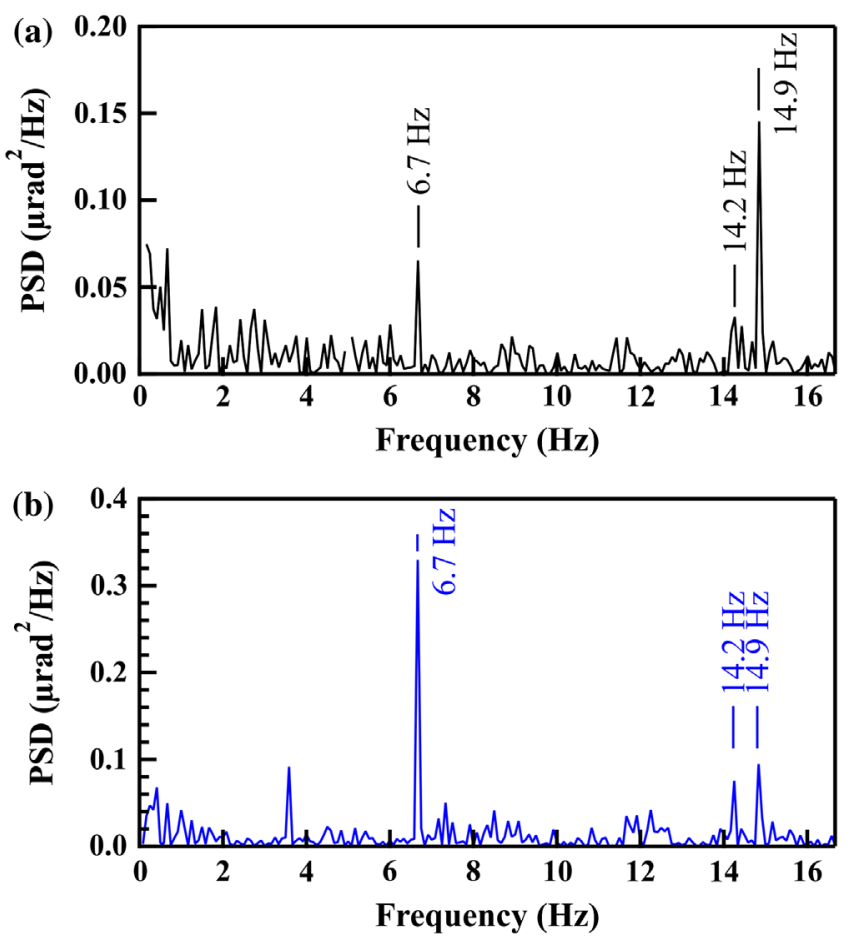

FIG. 5. PSD functions of the beam angle $y^{\prime}$ measured with (a) a Si (220) Laue single-crystal monochromator and (b) a Si (220) Bragg double-crystal monochromator at the barium $K$ edge. Note that the two measurements were performed on different dates with the two monochromators being on different supports located in different hutches. The Laue SCM was radiation cooled in air, while the Bragg DCM has a gravity-fed water cooling system. Therefore, the three frequency peaks marked on both (a) and (b) are not from the monochromator, which is the only optics in the experiment.

identified by analyzing both position contributions. This is another unique feature of the ps-BPM system.

To distinguish between election source vibration and that of monochromators, additional tests and analysis will be necessary, such as to evaluate the spectral frequencies of the system. In this work, to identify the origin of the beam motion shown in Fig. 3, measurements were carried out with two different monochromators: a Si (220) Bragg DCM at the barium $K$ edge and a $\mathrm{Si}$ (311) single Laue crystal monochromator at the iodine $K$ edge.

Apart from different measurement times, crystal geometry, and $K$-edge filter energies, the two monochromators had different mechanical designs and were mounted on different supporting structures and at different locations along the beam line. The cooling schemes of the two monochromators are also different. The Bragg DCM has a gravity-flow water cooling system, while the Laue SCM relied on the radiation cooling in air. Therefore, the two monochromators should not have the same vibration frequencies.

Figure 5 shows the PSD functions of $y^{\prime}$ obtained from the two separate experiments. Both results were extracted from 400 images with a sampling rate of $0.03 \mathrm{~s}$. Both PSD 
functions contain the $6.7,14.2$, and $14.9 \mathrm{~Hz}$ peaks, which is a clear indication that they are not from the monochromators but characteristic motions of the electron beam. The amplitudes of these three peaks are not identical, since they were not measured on the same day. The small peak around 3.6 Hz in Fig. 5(b) can be from either the DCM or the source.

Even though the data interpretation requires the understanding of the monochromator effects on the beam, the psBPM system still has the advantage in identifying vibration origins compared with other monochromator-required beam monitors, e.g., x-ray double-slit interferometry. From the beam-line optics point of view, the ps-BPM system can be used for monochromator diagnostics as well.

\section{Machine study example}

The ps-BPM system can provide direct measurements of source parameters, which is a promising tool for machine studies. The next example looks at the effects of the BMIT wiggler on the BM source as the superconducting wiggler field was changed. The experiment was performed at the iodine $K$ edge (33.169 keV) by the Si (220) DCM at the beam line. The iodine filter projected concentration was $60 \mathrm{mg} \mathrm{cm}^{-2}$. BM source parameters were measured at different magnetic fields of the BMIT wiggler.

The extracted electron source position, angular position, size, and divergence are shown in Fig. 6 as a function of the wiggler field. All results are an average of $24 \mathrm{~s}$. Varying the magnetic field of the wiggler alters the tune of the electron beam lattice, which changes source parameters at the BM location. The electron beam position and angle shift only slightly as the wiggler field changes as shown in Figs. 6(a) and 6(b), respectively.

Both the electron source size [Fig. 6(c)] and divergence [Fig. 6(d)] decrease as the wiggler field raises from 0 to $2 \mathrm{~T}$. The dramatic change in the source size and divergence is an indication of a significant lattice change. In fact, the BMIT wiggler is always required to operate above $2 \mathrm{~T}$ in order to keep the electron beam stable in the normal operation mode of the CLS. Results shown in Figs. 6(c) and 6(d) are direct proof of this strong lattice variation.

As the wiggler field raises above $2 \mathrm{~T}$, the source divergence becomes stable as shown in Fig. 6(d). The source size does vary a bit above $2 \mathrm{~T}$ but is comparable to the measurement error in this case [Fig. 6(c)].

Note that the results here are to show the sensitivity of the ps-BPM system, which is demonstrated by the clear detection of relative changes in all four quantities as shown in Fig. 6. The absolute electron source sizes in this study are different from the ones in Fig. 3, because they were measured at different times with different electron beam lattice parameters.

The ps-BPM system shows excellent sensitivity in measuring the source position, angle, and divergence but is less accurate in monitoring source sizes. However,
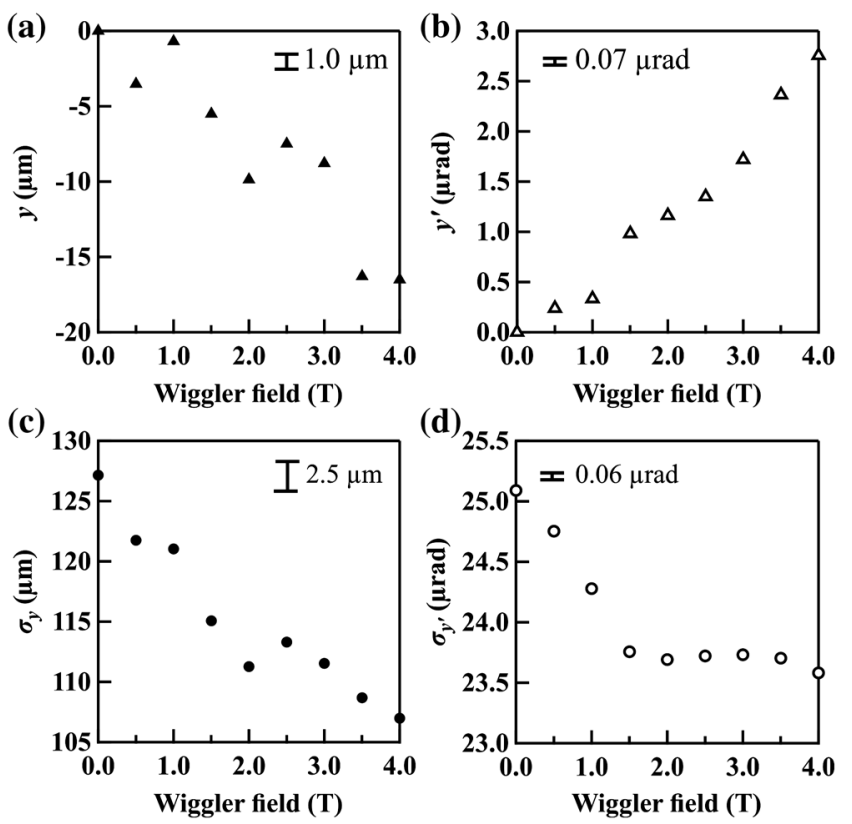

FIG. 6. Extracted (a) $y$, (b) $y^{\prime}$, (c) $\sigma_{y}$, and (d) $\sigma_{y}^{\prime}$ at the BMIT bend magnet as a function of the magnetic field of the BMIT wiggler. The error bars are the standard deviation of eight measurements of $3 \mathrm{~s}$ data.

simulation studies showed that by optimizing the system submicron sensitivity can be achieved, which is required for measuring sources sizes of a few microns [17].

\section{CONCLUSION}

We have shown how to use the ps-BPM system to characterize source properties. The capability of monitoring the source position, angular position, size, and divergence simultaneously is the unique feature of the ps-BPM system. Combining the time and frequency domain studies, the monitor can provide more systematic information about the source and the beam line. The full analysis of the system can be performed in real time owing to the simple physical model based on Gaussian distribution profiles.

The system was used during (i) normal operations where beam motions and size variations were monitored, (ii) changing the monochromator type to investigate sources of motions, and (iii) while an insertion device field was changed to assess the impact on electron beam motion and source size. In all instances, the system demonstrated its ability to provide useful information which is not possible by other means.

The ps-BPM system relies on a mechanically and thermally stable monochromator to provide accurate source measurements. The stability requirement of the monochromator is achievable with commercially available solutions. The stability can be enhanced by a specific monochromator design to this application such as the pseudo-channel-cut monochromator used in most of the experiments presented or a true channel-cut monochromator. In this instance, 
having relative motion between the two crystals is very much reduced and leaves only the easily detected case where both crystals move in angle, and steps can be taken to mitigate this.

The main purpose of this work is also to explore potential applications of the ps-BPM system. As lowemittance storage rings are being proposed and built, dedicated source diagnostic beam lines are a must. The ps-BPM system can be of particular interest for these new facilities and existing facilities under major upgrade. Results of this work will help the implementation and design of a practical ps-BPM system.

\section{ACKNOWLEDGMENTS}

The authors acknowledge the financial support of Natural Sciences and Engineering Research Council of Canada (NSERC) Discovery Grant, Canadian Institutes of Health Research (CIHR) Team Grant-Synchrotron Medical Imaging, CIHR Training Grant-Training in Health Research Using Synchrotron Techniques, Canada Research Chair Program, Saskatchewan Health Research Foundation Team Grant, and the University of Saskatchewan. The research described in this paper was performed at the Canadian Light Source, which is funded by the Canada Foundation for Innovation, NSERC, the National Research Council Canada, CIHR, the Government of Saskatchewan, Western Economic Diversification Canada, and the University of Saskatchewan. This work was also supported by the U.S. Department of Energy, Office of Basic Energy Sciences, under Contract No. DEAC02-06CH11357.

[1] P. F. Tavares, S. C. Leemann, M. Sjostrom, and A. Andersson, The MAX IV storage ring project, J. Synchrotron Radiat. 21, 862 (2014).

[2] M. Eriksson, J. F. van der Veen, and C. Quitmann, Diffraction-limited storage rings-A window to the science of tomorrow, J. Synchrotron Radiat. 21, 837 (2014).

[3] P. Elleaume, C. Fortgang, C. Penel, and E. Tarazona, Measuring beam sizes and ultra-small electron emittances using an X-ray pinhole camera, J. Synchrotron Radiat. 2, 209 (1995).

[4] C. Thomas, G. Rehm, I. Martin, and R. Bartolini, X-ray pinhole camera resolution and emittance measurement, Phys. Rev. Accel. Beams 13, 022805 (2010).

[5] T. Renner, H. Padmore, and R. Keller, Design and performance of the ALS diagnostic beamline, Rev. Sci. Instrum. 67, 3368 (1996).

[6] T. Weitkamp, O. Chubar, M. Drakopoulos, I. Snigireva, A. Snigirev, C. Schroer et al., Electron beam size and profile measurements with refractive X-ray lenses, in Proceedings of the European Particle Accelerator Conference, Vienna, 2000 (EPS, Geneva, 2000), pp. 1824-1826.

[7] K. Iida, N. Nakamura, H. Sakai, K. Shinoe, H. Takaki, M. Fujisawa et al., Measurement of an electron-beam size with a beam profile monitor using Fresnel zone plates, Nucl. Instrum. Methods Phys. Res., Sect. A 506, 41 (2003).

[8] S. Takano, M. Masaki, and H. Ohkuma, X-ray imaging of a small electron beam in a low-emittance synchrotron light source, Nucl. Instrum. Methods Phys. Res., Sect. A 556, 357 (2006).

[9] T. Mitsuhashi, Beam Measurement: Proceedings of the Joint US-CERN-Japan-Russia School on Particle Accelerators, Montreux, and CERN (World Scientific, Singapore, 1999).

[10] T. Naito and T. Mitsuhashi, Very small beam-size measurement by a reflective synchrotron radiation interferometer, Phys. Rev. Accel. Beams 9, 122802 (2006).

[11] J. Corbett, X. Huang, C. Li, T. Mitsuhashi, J. Wu, Y. Xuet al., Transverse beam profiling and vertical emittance control with a double-slit stellar interferometer, in Proceedings of the 5th International Beam Instrumentation Conference (IBIC'16), Barcelona, Spain, 2016 (JACoW, Geneva, Switzerland, 2017), pp. 236-239.

[12] J. P. Guigay, S. Zabler, P. Cloetens, C. David, R. Mokso, and M. Schlenker, The partial Talbot effect and its use in measuring the coherence of synchrotron x-rays, J. Synchrotron Radiat. 11, 476 (2004).

[13] X. Shi, S. Marathe, M. J. Wojcik, N. G. Kujala, A. T. Macrander, and L. Assoufid, Circular grating interferometer for mapping transverse coherence area of x-ray beams, Appl. Phys. Lett. 105, 041116 (2014).

[14] Å. Andersson, M. Böge, A. Lüdeke, V. Schlott, and A. Streun, Determination of a small vertical electron beam profile and emittance at the Swiss Light Source, Nucl. Instrum. Methods Phys. Res., Sect. A 591, 437 (2008).

[15] N. Samadi, B. Bassey, M. Martinson, G. Belev, L. Dallin, M. de Jong, and D. Chapman, A phase-space beam position monitor for synchrotron radiation, J. Synchrotron Radiat. 22, 946 (2015).

[16] N. Samadi, X. Shi, L. Dallin, and D. Chapman, A real-time phase-space beam emittance monitoring system, J. Synchrotron Radiat. 26, 1213 (2019).

[17] N. Samadi, X. Shi, and D. Chapman, Optimization of a phase-space beam position and size monitor for lowemittance light sources, J. Synchrotron Radiat. 26, 1863 (2019).

[18] N. Samadi, X. Shi, L. Dallin, and D. Chapman, A real-time phase-space beam emittance monitoring system, J. Synchrotron Radiat. 26, 1213 (2019).

[19] Y. A. Babanov, A. V. Ryazhkin, A. F. Sidorenko, and L. A. Blaginina, Correcting an experimental absorption spectrum for the core level width, J. Struct. Chem. 39, 833 (1998).

[20] W. H. Zachariasen, A general theory of x-ray diffraction in crystals, Acta Crystallogr. 23, 558 (1967).

[21] J. Schwinger, On the Classical Radiation of Accelerated Electrons, Phys. Rev. 75, 1912 (1949).

[22] T. W. Wysokinski, D. Chapman, G. Adams, M. Renier, P. Suortti, and W. Thomlinson, Beamlines of the biomedical imaging and therapy facility at the Canadian Light Source-Part 2, J. Phys. Conf. Ser. 425, 072013 (2013).

[23] T. W. Wysokinski, D. Chapman, G. Adams, M. Renier, P. Suortti, and W. Thomlinson, Beamlines of the biomedical 
imaging and therapy facility at the Canadian light sourcePart 1, Nucl. Instrum. Methods Phys. Res., Sect. A 582, 73 (2007).

[24] T. W. Wysokinski, D. Chapman, G. Adams, M. Renier, P. Suortti, and W. Thomlinson, Beamlines of the biomedical imaging and therapy facility at the Canadian light sourcePart 3, Nucl. Instrum. Methods Phys. Res., Sect. A 775, 1 (2015).
[25] L. Rebuffi and M. Sanchez del Río ShadowOui: A new visual environment for $\mathrm{x}$-ray optics and synchrotron beamline simulations, J. Synchrotron Radiat. 23, 1357 (2016).

[26] L. Rebuffi and M. Sanchez del Rio, OASYs (OrAnge SYnchrotron Suite): An open-source graphical environment for x-ray virtual experiments, Proc. SPIE 10388, 103880S (2017). 